\title{
Main scientific results of the DAMPE mission
}

\author{
Paolo Bernardinin ${ }^{1,2, a}$, on behalf of the DAMPE Collaboration \\ ${ }^{1}$ Dipartimento di Matematica e Fisica, Università del Salento, Lecce, Italy \\ ${ }^{2}$ Istituto Nazionale di Fisica Nucleare, Sezione di Lecce, Lecce, Italy
}

\begin{abstract}
DAMPE (DArk Matter Particle Explorer) is a satellite-born experiment, resulting from the collaboration of Chinese, Italian, and Swiss institutions. Since December 2015, DAMPE flights at the altitude of $500 \mathrm{~km}$ and collects data smoothly. The detector is made of four sub-detectors: top layers of plastic scintillators, a silicon-tungsten tracker, a BGO calorimeter (32 radiation lengths), and a bottom boron-doped scintillator to detect delayed neutrons. The main goal of the experiment is the search for indirect signals of Dark Matter in the electron and photon spectra with energies up to $10 \mathrm{TeV}$. Furthermore DAMPE studies cosmic charged and gamma radiation. The calorimeter depth and the large acceptance allow to measure cosmic ray fluxes in the range from $20 \mathrm{GeV}$ up to hundreds of TeV. An overview of the latest results about light component $(\mathrm{p}+\mathrm{He})$ of charged cosmic rays, gamma astronomy and electron and positron spectrum will be presented.
\end{abstract}

\section{The detector}

The DArk Matter Particle Explorer (DAMPE) is a satellite-born experiment and it is funded by the strategic space projects of the Chinese Academy of Sciences with the contribution of Italian and Swiss agencies [1, 2]. It is devoted to measure the fluxes of charged cosmic rays (electrons, protons and heavier nuclei), to study the high energy gamma signal of astrophysical origin and to search for indirect dark matter signatures.

The detector design was performed looking for the proper compromise between research goals and limitations on geometry, power consumption and weight. In Fig. 1 the four sub-detectors of the DAMPE experiment are shown:

- PSD, in place of Plastic Scintillator strip Detector, used to measure the charge (Z) of cosmic rays and to select gamma events (events in anti-coincidence with the PSD signal),

- STK, in place of Silicon-Tungsten tracKer-converter, to reconstruct the trajectory of incident particles, to repeat the charge measurement and to convert photons in electron-positron pairs,

- BGO, in place of bismuth germanate imaging calorimeter (14 layers) of about 32 radiation lengths, to reach high resolution in energy measurement and to distinguish between hadronic and electromagnetic showers,

- NUD, in place of NeUtron Detector, to further increase the hadronic shower rejection power.

ae-mail: paolo.bernardini@le.infn.it 


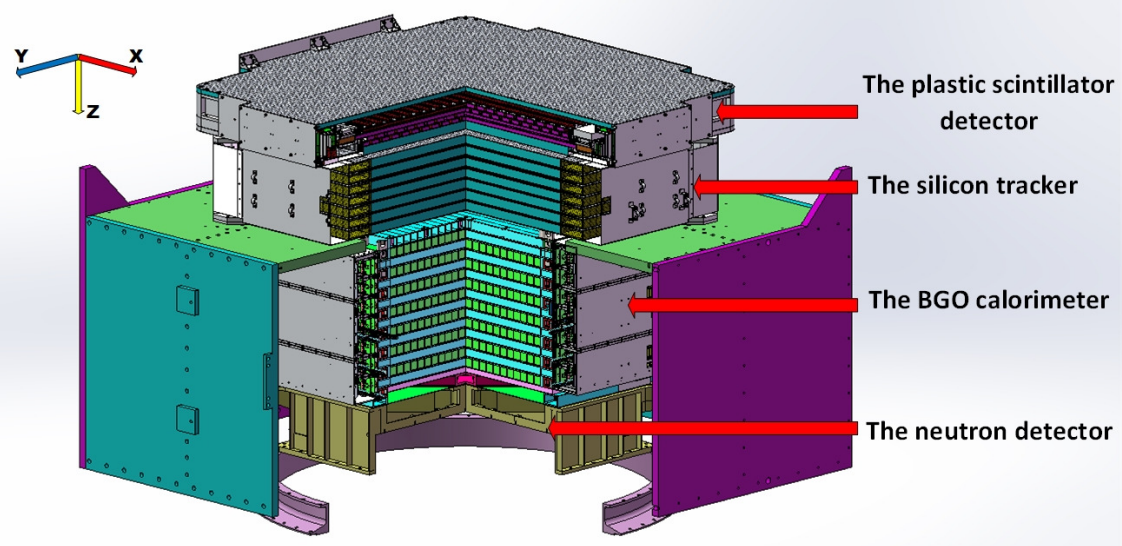

Figure 1. The DAMPE detector

DAMPE performances were verified by a series of beam tests at CERN [1, 3, 4]. The wide energy range of electron beam and the high purity of proton beam allowed to check energy resolution, linearity (Fig. 2) and $e / p$ separation (see Fig. 8 in the following). Also beams of argon and lead fragments were used to check the PSD capability to measure $\mathrm{Z}$ for heavy ions (Fig. 3 for the argon beam). Furthermore sea-level muon test have been performed during different stages of the DAMPE assembly. Then the energy response to Minimum Ionizing Particles (MIPs), the efficiency and the detector alignment have been checked with a large sample of cosmic ray muon events.

The satellite has been successfully put on orbit on December 17, 2015. After few days all detectors were powered on and the data acquistion began very soon. Presently DAMPE flies at the altitude of $500 \mathrm{~km}$ on a Sun-synchronous orbit.

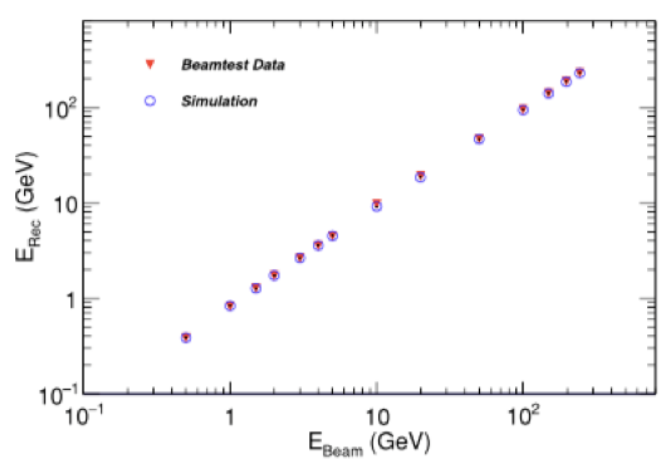

Figure 2. Beam test - Reconstructed energy versus electron beam energy.

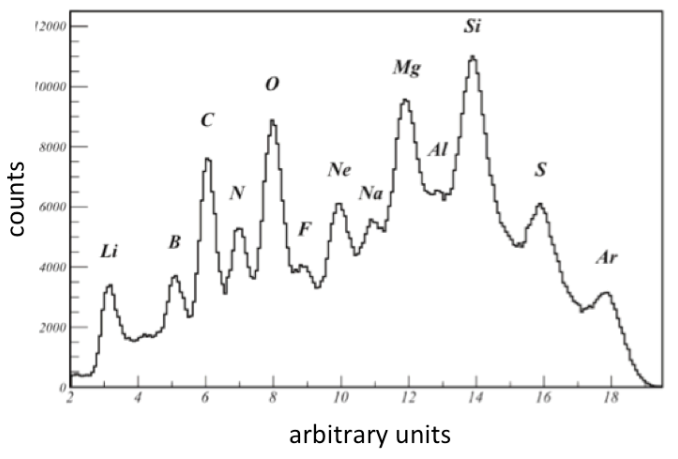

Figure 3. Beam test - PSD charge spectrum of fragments of an argon beam (helium peak has been removed). 
On orbit the detector is calibrated by using the standard scientific data and dedicated calibration data. The data acquisition system runs continuously in observation mode for most of the time and switches to calibration mode for 40 seconds twice per orbit. All the calibration results (pedestal values, dynode ratios, energy gains, trigger thresholds and so on) are consistent with the ground ones and very stable after temperature correction. The STK alignment allows a significant improvement of the tracking resolution [5]. In Fig. 4 (left panel) the PSD signal due to minimum ionizing protons is plotted as a function of time in the period December 2016 - March 2017. A similar plot is shown in the right panel of Fig. 4 for BGO signal due to helium nuclei not showering in the BGO calorimeter. In both cases the detector response is very stable.
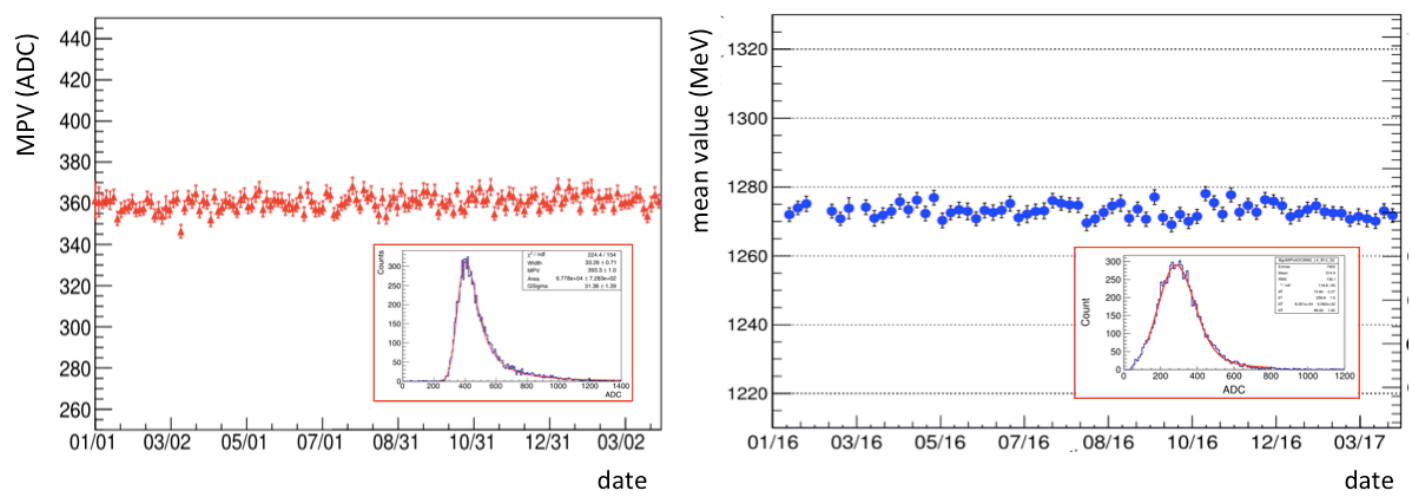

Figure 4. Left panel: the deposited energy in PSD for minimum ionizing protons is fitted with the convolution of a Landau function with a Gaussian one (insert). The Most Probable Value (MPV) s shown as a function of time. Right panel: similar plot for minimum ionizing helium nuclei in BGO (the mean value of the deposited energy is displayed versus time instead of MPV).

\section{Cosmic rays}

The cosmic ray (CR) studies are based on the charge measurement (square root of the energy release in PSD is proportional to Z), the STK tracking and the BGO imaging. The event shape in the calorimeter allows to distinguish electromagnetic showers from hadronic ones. Furthermore the unprecedented thickness of the calorimeter allows to push the direct measurement of CR spectra to highest energies with excellent energy resolution. Indeed DAMPE CR measurements are going to reach energies of some hundreds of $\mathrm{TeV}$.

The template fit for protons and helium in Fig. 5 shows an excellent data-Montecarlo agreement and allows to estimate the contamination in each peak. The preliminary measurement of proton and helium fluxes are presented in Fig. 6.

\section{Gamma astronomy}

Another aim of the DAMPE mission is the gamma astronomy by collecting events without hits on the PSD layers. In less than 1 year DAMPE is able to scan the whole sky by looking for bright gamma sources, detecting their fluxes and possible variability. The gamma periodicity of two brightest pulsars (Geminga and Vela) has been measured in the energy range from $1 \mathrm{GeV}$ to $100 \mathrm{GeV}$ [6]. In Fig. 7 the flux of the variable high energy gamma emission detected by DAMPE from two active galactic nuclei (CTA102, 3C454.3) is shown [7]. 


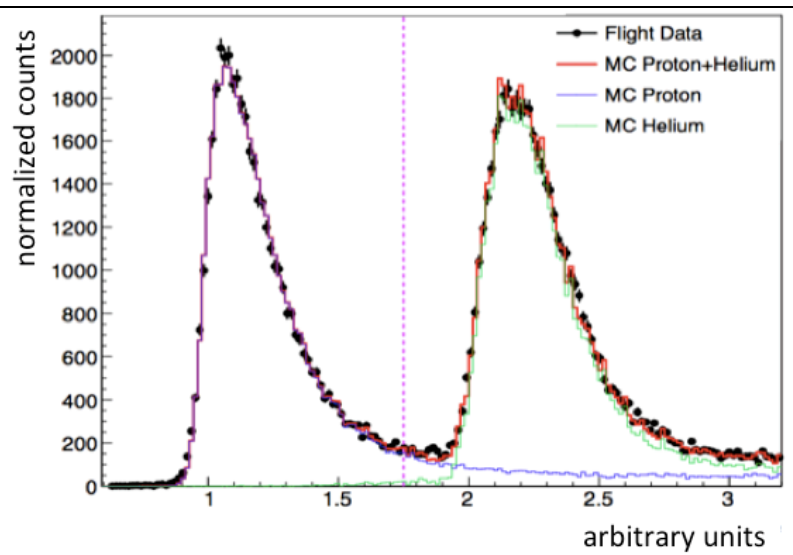

Figure 5. Combined PSD signal for protons and helium nuclei, for BGO energies in the range from $447 \mathrm{GeV}$ to $562 \mathrm{GeV}$. The color codes to distinguish on-orbit and simulated data are shown in the plot. The cut to select proton candidates is represented by the vertical dotted line.
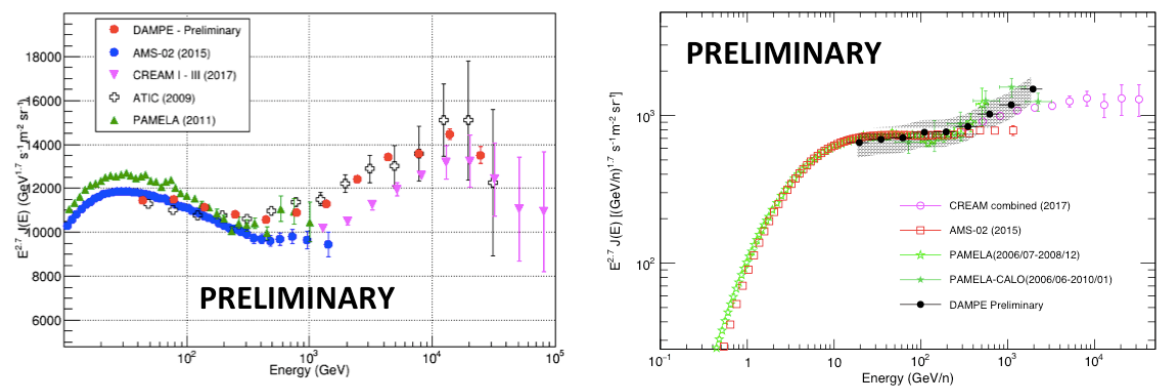

Figure 6. Spectra of protons (left panel) and helium nuclei (right panel).
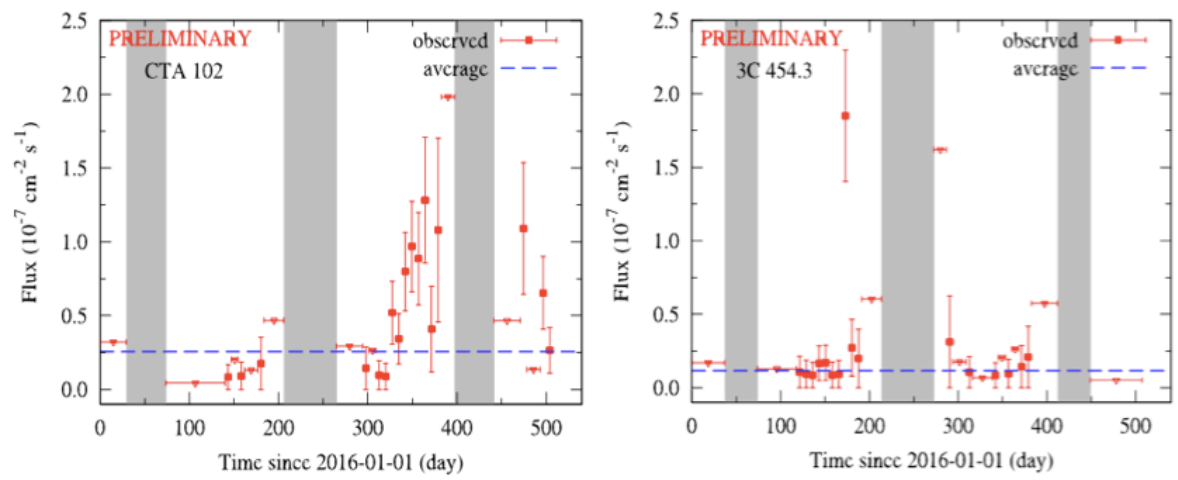

Figure 7. The light curves for two active galactic nuclei (left: CTA 102, right: 3C454.3). 


\section{Measurement of electron and positron spectrum}

In the last years some measurements [8-14] reported an unexpected shape of the flux for CR electrons and positrons (CRE). Furthermore evidence for a spectral break in the TeV range of the CRE flux has been provided by indirect measurements $[15,16]$, although the results were affected by significant uncertainties.

In around two years of data taking DAMPE made a direct measurement of CREs in the range from $25 \mathrm{GeV}$ to $4.6 \mathrm{TeV}$ [17] with unprecedented accuracy (high energy resolution and low background). Indeed the depth and the imaging properties of the BGO calorimeter are exploited to recognise the showers initiated by CRE. The identification algorithm is based on the spread of the BGO hits (spread) and on the fraction $\left(F_{\text {last }}\right)$ of the energy on the lower layer with respect to the sum of energies released on all the BGO layers. The quantities spread and $F_{\text {last }}$ are combined in the parameter

$$
\zeta=F_{\text {last }} \frac{(\text { spread } / \mathrm{mm})^{4}}{8 \times 10^{6}} .
$$

The efficiency of the $\zeta$-parameter in order to distinguish electrons and protons has been verified in the beam test (Fig. 8). Then the CRE sample is selected by requiring $\zeta<8.5$ (see Fig. 9, left panel). The CRE flux measured by DAMPE as a function of energy is shown in right panel of Fig. 9 with measurements by others experiments. A smoothly broken power law is preferred to fit the largest part of the spectrum instead of a single power law model. More details are available in [17].
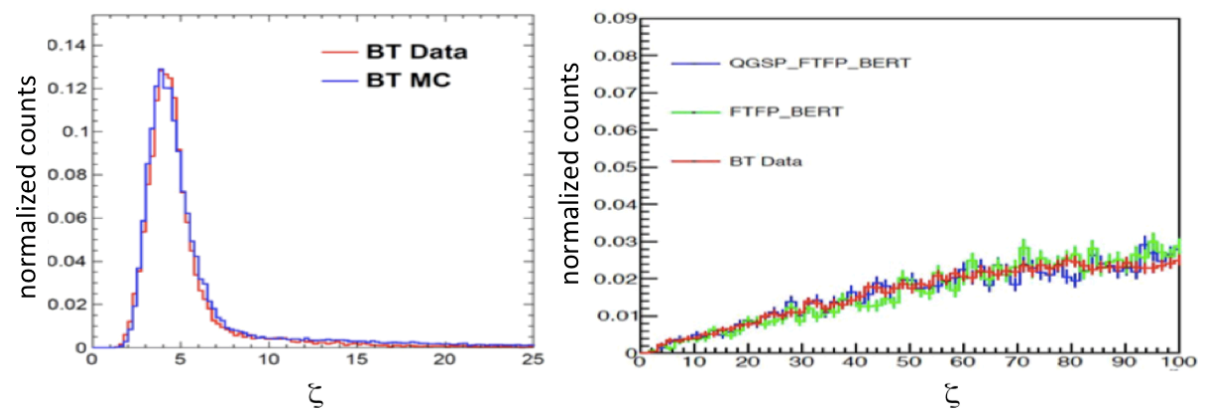

Figure 8. Beam test - The $\zeta$-distributions for electrons (left panel) and for protons (right panel). In the proton plot the real data are compared with simulations based on different models of the hadronic interactions.

\section{Conclusions}

The DAMPE detector works extremely well since its launch more than 2.5 years ago and it is expected to operate stably in the next few years. Many analyses based on DAMPE data are going on successfully on cosmic rays and gamma astronomy. The measurement of CRE spectrum is particularly remarkable. Indeed the direct detection of a spectral break at $\sim 0.9 \mathrm{TeV}$ confirms what found by previous indirect measurements, clarifies the shape of the CRE spectrum at energies above $1 \mathrm{TeV}$ and sheds light on the physical origin of the sub-TeV CREs. 

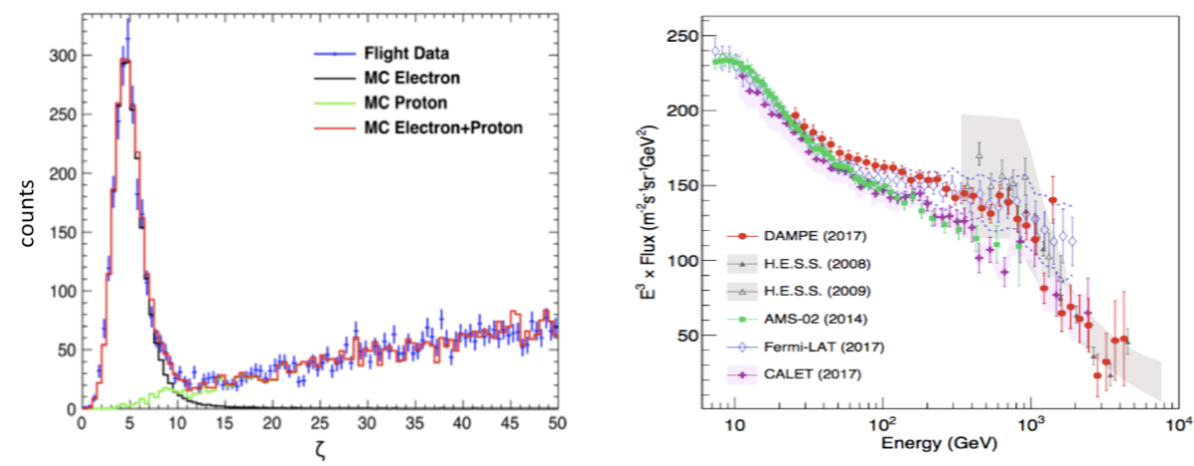

Figure 9. Left: Distribution of the $\zeta$ parameter (the cut $\zeta<8.5$ is applied to select the CRE sample). Right: electron and positron spectrum measured by different experiments (DAMPE: red dots, HESS systematic errors: grey band).

\section{References}

[1] Chang J., Chinese J. Space Science 34, 550 (2014)

[2] Chang J. et al., Astropart. Physics 95, 6 (2017)

[3] Azzarello P. et al., Nuclear Instruments and Methods A 831, 378 (2016)

[4] Zhang Z. et al., Nuclear Instruments and Methods A 836, 98 (2016)

[5] Tykhonov A. et al., Nuclear Instruments and Methods A 893, 43 (2018)

[6] Salinas M.M. et al., PoS(ICRC2017) 709 (2017)

[7] Yuan Q. et al., PoS(ICRC2017) 617 (2017)

[8] Chang J. et al. (The ATIC Collaboration), Nature 456, 362 (2008)

[9] Abdo A.A. et al. (The Fermi-LAT Collaboration), Phys. Rev. Lett. 102, 181101 (2009)

[10] Abdollahi S. et al. (The Fermi-LAT Collaboration), Phys. Rev. D 95, 082007 (2017)

[11] Aguilar M. et al. (The AMS-02 Collaboration), Phys. Rev. Lett. 113, 221102 (2014)

[12] Adriani O. et al. (The PAMELA collaboration), Phys. Rev. Lett. 106, 201101 (2011)

[13] Adriani O. et al. (The CALET Collaboration), Phys. Rev. Lett. 119, 181101 (2017)

[14] Adriani O. et al. (The CALET Collaboration), Phys. Rev. Lett. 120, 261102 (2018)

[15] Aharonian F. et al. (The H.E.S.S. Collaboration), Phys. Rev. Lett. 101, 261104 (2008)

[16] Aharonian F. et al. (The H.E.S.S. Collaboration), Astron. Astrophys. 508, 561 (2009)

[17] Ambrosi G. et al. (The DAMPE Collaboration), Nature 552, 63 (2017) 